\title{
On Determination of Inter and Intrinsic Diffusion Coefficients in Binary System
}

\author{
By Masami Onishi*, Yoshinori Wakamatsu* \\ and Toshitada Shimozaki*
}

\begin{abstract}
The conceptions of various frames of reference for binary interdiffusion, which have been partly reported in the past, are summarized. The rational method of determining the inter- and intrinsic diffusion coefficients in the general case, where total volume of a diffusion couple changes during diffusion, is reviewed and discussed. The interdiffusion coefficients ordinarily defined are correctly calculated irrespective of reference frame. The magnitude of the intrinsic diffusion coefficients depends on what is chosen for the frame of reference. The useful intrinsic diffusion coefficient in alloys is the one relative to the number of mole fixed frame of reference.
\end{abstract}

(Received July 25, 1983)

Keyzords: diffusion in binary alloys, interdiffusion coefficients, intrinsic diffusivities, volume fixed frame, mass fixed frame, number of mole fixed frame, total volume change of diffusion couple, determination of various diffusivities

\section{Introduction}

For a binary system, in which total molar volume is kept constant, there are presumably two types of the diffusion fluxes, i.e., $-\tilde{D} \partial C_{i} / \partial x$ and $-D_{i} \partial C_{i} / \partial x$, of component $i(i=1,2)$ in a diffusion couple. On the contrary, one can suppose five types of the fluxes of component $i$, when total volume of a couple changes during diffusion (see section II). The five types have the distinct reference planes which are parallel to one another, and the flux across a certain type of reference plane is conventionally called the one relative to a certain frame of reference or a coordinate system.

When the flux relative to one type among five types of frames of reference and that relative to one type among the rest four are set to be ${ }_{\alpha} J_{i}$ and ${ }_{\beta} J_{i}$, respectively, ${ }_{\beta} J_{i}$ is linearly related to ${ }_{\alpha} J_{i}$ by the equation

$$
{ }_{\alpha} J_{i}={ }_{\beta} J_{i}+C_{i} \omega_{\beta \alpha}
$$

Here ${ }_{\alpha} J_{i}$ and ${ }_{\beta} J_{i}$ have a unit of (quantity of substance $/ \mathrm{m}^{2} \mathrm{~s}$ ), $C_{i}$ is in a unit of (quantity of substance $/ \mathrm{m}^{3}$ ) and $\omega_{\beta \alpha}$ is the velocity of the frame $\beta$ relative to the frame $\alpha$, where the quantity of substance may be either of the num-

* Metal Processing Engineering, The Kyushu Institute of Technology, Kitakyushu 804, Japan. ber of mole or the mass in $\mathrm{kg}$. If one chooses the flux relative to the plane fixed with respect to an inert marker in a diffusion zone for ${ }_{\beta} J_{i}$ in eq. (1), the magnitude of the moving velocity, $\omega_{\beta \alpha}$, of the marker depends on any choice of reference frame for $J_{i}$.

In our previous paper ${ }^{(1)}$ we defined three distinct types of the intrinsic diffusion coefficients, $D_{i}, D_{i}^{\prime}$ and $D_{i}^{\prime \prime}$, of component $i$, where they should be, respectively, called as the intrinsic ones relative to the volume, the number of mole and the mass fixed frames of reference. The main aims of this article are to define various frames of reference and their mutual relations which have been partly reported in the past and to discuss the rational method by which the interdiffusion coefficients and intrinsic diffusivities are determined.

\section{Frames of Reference and Flux Equations}

In this article the cross sectional area in $\mathrm{m}^{2}$ must be constant in a binary diffusion couple in which one-dimensional diffusion occurs, and the $x$ axis having an ordinary length unit in $\mathrm{m}$ is fixed with respect to the non-diffusion end of the couple at $x=-\infty$. The five types of fluxes which have the unit of $\mathrm{mol} / \mathrm{m}^{2} \mathrm{~s}$, are those measured relative to the volume, the number of mole, the mass and the cell fixed frames of reference as well as the fluxes relative to the 
marker fixed planes. Their definitions can be seen in what follows:

\section{Fluxes for the volume fixed frame of reference}

This type of fluxes is characterized by ${ }^{(2)}$

$$
\bar{V}_{1} J_{1}+\bar{V}_{2} J_{2}=0
$$

where

$$
{ }_{v} J_{i} \equiv-\widetilde{D} \frac{\partial C_{i}}{\partial x} \quad(i=1,2)
$$

and $\bar{V}_{i}(i=1,2)$ is the partial molar volume in the unit of $\mathrm{m}^{3} / \mathrm{mol} . C_{i}(i=1,2)$ is the molar concentration in the unit of $\mathrm{mol} / \mathrm{m}^{3}$, and $\widetilde{D}$ is the so called interdiffusion coefficient. As seen in eq. (3), this type of flux is given by Fick's first law, and its reference plane is not fixed with respect to the $x$ axis except for the case where the total molar volumes of alloys are expressed by a linear function of mole fraction. For the variable partial molar volumes, therefore, one can not designate the position of the reference plane of the flux on the $x$ axis.

\section{Fluxes for the number of mole fixed frame of reference}

Using eq. (4), we can derive eq. (5) from Fick's first law.

$$
\mathrm{d} C_{i}=N_{i} \mathrm{~d}(1 / V)+\mathrm{d} N_{i} / V \quad(i=1,2)
$$

and

$$
-\frac{\widetilde{D}}{V} \frac{\partial N_{i}}{\partial x}=-\widetilde{D} \frac{\partial C_{i}}{\partial x}+C_{i}\left\{V \widetilde{D} \frac{\partial(1 / V)}{\partial x}\right\} \quad(i=1,2) .
$$

Here $N_{i}$ is the mole fraction of component $i$, and $V$ is the total molar volume of alloys. The term on the left hand side in eq. (5) corresponds to the flux relative to this frame of reference or the center of moles ${ }^{(3)}$, being defined by

$$
{ }_{m} J_{1}+{ }_{m} J_{2}=0
$$

where

$$
{ }_{m} J_{i} \equiv-\frac{\tilde{D}}{V} \frac{\partial N_{i}}{\partial x} \quad(i=1,2) .
$$

The bracketed term in eq. (5) means the moving velocity of the volume fixed frame relative to the number of mole fixed frame, being given by

$$
V \tilde{D} \frac{\partial(1 / V)}{\partial x}=-\left(\bar{V}_{1} \frac{\tilde{D}}{V} \frac{\partial N_{1}}{\partial x}+\bar{V}_{2} \frac{\widetilde{D}}{V} \frac{\partial N_{2}}{\partial x}\right) .
$$

\section{Fluxes for the mass fixed frame of reference}

For example, the flux across the plane fixed with respect to the center of gravity of a diffusion couple is that for the mass fixed frame of reference. The relation between molar concentration, $C_{i}$, and mass fraction, $W_{i}$, is as follows:

$$
C_{i}=\frac{\rho}{M_{i}} W_{i} \quad(i=1,2),
$$

where $\rho$ is the density of alloys and $M_{i}$ is the atomic weight of component $i(i=1,2)$. Differentiation of eq. (9) yields

$$
\mathrm{d} C_{i}=\frac{\rho}{M_{i}} \mathrm{~d} W_{i}+\frac{W_{i}}{M_{i}} \mathrm{~d} \rho \quad(i=1,2) .
$$

Expanding the flux given by Fick's first law with the aid of eq. (10), one obtains

$$
-\frac{\rho}{M_{i}} \widetilde{D} \frac{\partial W_{i}}{\partial x}=-\widetilde{D} \frac{\partial C_{i}}{\partial x}+C_{i} \frac{\tilde{D}}{\rho} \frac{\partial \rho}{\partial x} \quad(i=1,2) .
$$

The flux for this frame of reference is given by the term on the left hand side of eq. (11), being characterized by

$$
M_{1 w} J_{1}+M_{2 w} J_{2}=0,
$$

where

$$
{ }_{w} J_{i} \doteq-\frac{\rho}{M_{i}} \widetilde{D} \frac{\partial W_{i}}{\partial x} \quad(i=1,2)
$$

\section{Fluxes for the cell fixed frame of reference}

This type of flux was first derived by Prager ${ }^{(4)}$, and the flux was named that for the cell fixed frame of reference by Kirkwood et al. ${ }^{(5)}$, being related to Fick's first law by the equation

$$
\begin{array}{r}
J_{i}=-\widetilde{D} \frac{\partial C_{i}}{\partial x}+C_{i} \int_{-\infty}^{x} \frac{\tilde{D}}{\bar{V}_{2} C_{1}} \frac{\partial \bar{V}_{2}}{\partial C_{1}}\left(\frac{\partial C_{1}}{\partial x}\right)^{2} \mathrm{~d} x \\
(i=1,2) . \quad(14)
\end{array}
$$

Balluffi $^{(6)}$ introduced the following form for ${ }_{c} J_{i}$ in eq. (14)

$$
{ }_{c} J_{i} \equiv-\theta_{i} \frac{\partial C_{i}}{\partial x} \quad(i=1,2)
$$

and also defined $\theta_{i}$ by 


$$
\theta_{i}=D_{i}-U C_{i} \frac{\partial x}{\partial C_{i}} \quad(i=1,2)
$$

where $D_{i}$ is the intrinsic diffusivity of component $i$ relative to the volume fixed frame of reference (see next paragraph) and $U$ is the marker velocity relative to the $x$ axis. For the variable partial molar volumes the reference planes of this frame of reference are fixed with respect to the $x$ axis, and the reference planes of the volume fixed frame move relative to the $x$ axis with the velocity given by the integrand in eq. (14). If the total molar volumes of alloys are expressed by a linear function of mole fraction, this frame coincides with the volume fixed frame.

\section{Fluxes across the planes fixed with respect to markers (intrinsic fluxes)}

Since Darken ${ }^{(7)}$ introduced the conception of the intrinsic fluxes, the representation of $-D_{i} \partial C_{i} / \partial x$ is conventionally used for the fluxes. However, there are other representations for the fluxes, and they are given in terms of $-\left(D_{i}^{\prime} / V\right)\left(\partial N_{i} / \partial x\right)^{(1)(8)}$ and $-\left(\rho D_{i}^{\prime \prime} / M_{i}\right)\left(\partial W_{i} /\right.$ $\partial x)^{(1)}$ which have the same unit $\left(\mathrm{mol} / \mathrm{m}^{2} \mathrm{~s}\right)$ and magnitude as $-D_{i} \partial C_{i} / \partial x$ have.

When the moving velocities of a marker relative to the volume, the number of mole and the mass fixed frames of reference are, respectively, given by $u, u^{\prime}$ and $u^{\prime \prime}$, the following equations are obtained.

$$
\begin{aligned}
& -\tilde{D} \frac{\partial C_{i}}{\partial x}=-D_{i} \frac{\partial C_{i}}{\partial x}+C_{i} u \quad(i=1,2) \\
& -\frac{\tilde{D}}{V} \frac{\partial N_{i}}{\partial x}=-\frac{D_{i}^{\prime}}{V} \frac{\partial N_{i}}{\partial x}+C_{i} u^{\prime} \quad(i=1,2)
\end{aligned}
$$

and

$$
-\frac{\rho \tilde{D}}{M_{i}} \frac{\partial W_{i}}{\partial x}=-\frac{\rho D_{i}^{\prime \prime}}{M_{i}} \frac{\partial W_{i}}{\partial x}+C_{i} u^{\prime \prime} \quad(i=1,2),
$$

where

$$
\begin{aligned}
& u=\bar{V}_{1}\left(D_{1}-D_{2}\right) \frac{\partial C_{1}}{\partial x} \\
& u^{\prime}=\left(D_{1}^{\prime}-D_{2}^{\prime}\right) \frac{\partial N_{1}}{\partial x}
\end{aligned}
$$

and

$$
u^{\prime \prime}=\left(D_{1}^{\prime \prime}-D_{2}^{\prime \prime}\right) \frac{\partial W_{1}}{\partial x}
$$

Here $D_{i}, D_{i}^{(1)(8)}$ and $D_{i}^{\prime(1)}$ may be called the intrinsic diffusivities relative to the volume, the number of mole and the mass fixed frames of reference, respectively. In order to obtain the relation between $D_{i}$ and the other types of the intrinsic diffusivities, setting

$$
\begin{aligned}
j_{i} & \equiv-D_{i} \frac{\partial C_{i}}{\partial x}=-\frac{D_{i}^{\prime}}{V} \frac{\partial N_{i}}{\partial x} \quad(i=1,2) \\
& =-\frac{\rho D_{i}^{\prime \prime}}{M_{i}} \frac{\partial W_{i}}{\partial x} \quad(i=1,2)
\end{aligned}
$$

and utilizing eq. $(25)^{(3)}$ as well as eq. (26) [see appendix for derivation of eq. (26)]

$$
\mathrm{d} C_{1}=\frac{\bar{V}_{2}}{V^{2}} \mathrm{~d} N_{1} ; \mathrm{d} C_{2}=\frac{\bar{V}_{1}}{V^{2}} \mathrm{~d} N_{2}
$$

and

$$
\mathrm{d} C_{1}=\frac{\bar{V}_{2} \rho^{2}}{M_{1} M_{2}} \mathrm{~d} W_{1} ; \mathrm{d} C_{2}=\frac{\bar{V}_{1} \rho^{2}}{M_{1} M_{2}} \mathrm{~d} W_{2},
$$

one obtains

$$
D_{1}^{\prime}=\frac{D_{1} \bar{V}_{2}}{V} ; D_{2}^{\prime}=\frac{D_{2} \bar{V}_{1}}{V}
$$

and

$$
D_{1}^{\prime \prime}=\frac{D_{1} \bar{V}_{2} \rho}{M_{2}} ; D_{2}^{\prime \prime}=\frac{D_{2} \bar{V}_{1} \rho}{M_{1}} .
$$

If the density, $\rho$, of the alloys does not depend on the composition, three types of the intrinsic diffusivities of component $i$ are equivalent to one another.

\section{Useful Relations Derived from Flow Equations}

Because the fluxes of ${ }_{m} J_{i},{ }_{w} J_{i},{ }_{c} J_{i}$ and $j_{i}$ are related to those for the volume fixed frame of reference, as shown by eqs. (5), (11), (14) and (17), it is clear that eq. (1) holds for the frames of reference under consideration. When $\omega_{\beta \alpha}$ is eliminated from two eqs. (1) as to components 1 and 2 , it follows that

$$
C_{2 \alpha} J_{1}-C_{1 \alpha} J_{2}=C_{2 \beta} J_{1}-C_{1 \beta} J_{2} .
$$

Relations between the various types of fluxes, $J_{i}$, and $\left(C_{2} J_{1}-C_{1} J_{2}\right)$ are summarized in Table 1. All terms of $\left(C_{2} J_{1}-C_{1} J_{2}\right)$ in the second column in Table 1 are equivalent to one another, so that the following important relations are derived: 
Table 1 Relation between $J_{i}$ and $\left(C_{2} J_{1}-C_{1} J_{2}\right)$.

\begin{tabular}{|c|c|c|c|}
\hline & $\begin{array}{l}J_{i}(i=1,2) \\
\left(\mathrm{mol} / \mathrm{m}^{2} \mathrm{~s}\right)\end{array}$ & & $\begin{array}{l}C_{2} J_{1}-C_{1} J_{2} \\
\left(\mathrm{~mol}^{2} / \mathrm{m}^{5} \mathrm{~s}\right)\end{array}$ \\
\hline 1 & $-\tilde{D} \frac{\partial C_{t}}{\partial x} \equiv_{v} J_{t}$ & & $-\frac{\tilde{D}}{\bar{V}_{2}} \frac{\partial C_{l}}{\partial x}$ \\
\hline 2 & $-\frac{\tilde{D}}{V} \frac{\partial N_{t}}{\partial x} \equiv_{m} J_{t}$ & & $-\frac{\tilde{D}}{V^{2}} \frac{\partial N_{1}}{\partial x}$ \\
\hline 3 & $-\frac{\rho \widetilde{D}}{M_{i}} \frac{\partial W_{l}}{\partial x} \equiv_{w}$ & & $-\frac{\rho^{2} \tilde{D}}{M_{1} M_{2}} \frac{\partial W_{1}}{\partial x}$ \\
\hline 4 & $-\theta_{t} \frac{\partial C_{i}}{\partial x} \equiv_{c} J_{i}$ & & $-\frac{C_{2} \bar{V}_{2} \theta_{1}+C_{1} \bar{V}_{1} \theta_{2}}{\bar{V}_{2}} \frac{\partial C_{1}}{\partial x}$ \\
\hline $5 a$ & $-D_{t} \frac{\partial C_{i}}{\partial x}$ & & $-\frac{C_{2} \bar{V}_{2} D_{1}+C_{1} \bar{V}_{1} D_{2}}{\bar{V}_{2}} \frac{\partial C_{1}}{\partial x}$ \\
\hline $5 b$ & $-\frac{D_{l}^{\prime}}{V} \frac{\partial N_{t}}{\partial x}$ & $\equiv j_{i}$ & $-\frac{N_{2} D_{1}^{\prime}+N_{1} D_{2}^{\prime}}{V^{2}} \frac{\partial N_{1}}{\partial x}$ \\
\hline $5 \mathrm{c}$ & $-\frac{\rho D_{t}^{\prime \prime}}{M_{t}} \frac{\partial W_{i}}{\partial x}$ & & $-\frac{\rho^{2}\left(W_{2} D_{1}^{\prime \prime}+W_{1} D_{2}^{\prime \prime}\right)}{M_{1} M_{2}} \frac{\partial W_{1}}{\partial x}$ \\
\hline
\end{tabular}

$$
\begin{aligned}
\tilde{D} & =C_{1} \bar{V}_{1} \theta_{2}+C_{2} \bar{V}_{2} \theta_{1} \\
& =C_{1} \bar{V}_{1} D_{2}+C_{2} \bar{V}_{2} D_{1} \\
& =N_{1} D_{2}^{\prime}+N_{2} D_{1}^{\prime} \\
& =W_{1} D_{2}^{\prime \prime}+W_{2} D_{1}^{\prime \prime} .
\end{aligned}
$$

For any arbitrary frame of reference one may write the diffusion flux as $C_{i} v_{i}{ }^{(9)}$, where $v_{i}$ is the local velocity of component $i(i=1,2)$. If one type of the fluxes among five types in Table 1 is chosen for $C_{i} v_{i}$, we obtain

$$
C_{1} v_{1}=J_{1}+C_{1} \omega
$$

and

$$
C_{2} v_{2}=J_{2}+C_{2} \omega
$$

These equations yield

$$
C_{1} C_{2}\left(v_{1}-v_{2}\right)=C_{2} J_{1}-C_{1} J_{2} .
$$

Because the term on the right hand side in eq. (36) can be chosen from the second column in Table 1, one obtains the following equations which have been presented by Wagner ${ }^{(10)}$ in order to define $\tilde{D}$.

$$
C_{1} C_{2}\left(v_{1}-v_{2}\right)=-\frac{\tilde{D}}{V^{2}} \frac{\partial N_{1}}{\partial x}
$$

and

$$
\tilde{D}=\frac{N_{1} N_{2}\left(v_{1}-v_{2}\right)}{\partial N_{2} / \partial x}=\frac{V\left(N_{2} J_{1}-N_{1} J_{2}\right)}{\partial N_{2} / \partial x} .
$$

Stark $^{(9)}$ stated the invariance of $\tilde{D}$ from the viewpoint of the invariability of $\left(v_{1}-v_{2}\right)$ regardless of the frame of reference, but his statement is valid for the only case when eq. (1) holds for the frames of reference under consideration.

\section{Determination of Diffusion Fluxes and Diffusion Coefficients}

\section{Use of ordinary laboratory frame of reference}

By employing an electron probe microanalyzer one can construct the penetration curves in terms of the $N_{i}-x$ or $W_{i}-x$ curves, and then $C_{i}-x$ curves are determined by knowing the molar volume, $V$, of alloys as a function of composition. For the purpose of obtaining the diffusion fluxes the penetration curves must be integrated, so that the following initial conditions are introduced:

and

$$
C_{i}=C_{i}^{-}, \quad x<x_{0}, \quad t=0
$$

$$
C_{i}=C_{i}^{+}, \quad x>x_{0}, \quad t=0,
$$

where $x_{0}$ is fixed with respect to the nondiffusion end of a couple at $x=-\infty$ for all $t$. In the systems involving a change in volume upon mixing, the two ends of a diffusion couple move with respect to each other. Consequently, the $x_{0}^{\prime}$ plane fixed with respect to the nondiffusion end of the diffusion couple at $x=\infty$ moves with respect to the $x_{0}$ plane defined above. At a given diffusion time the product of $\left(x_{0}^{\prime}-x_{0}\right)$ times the sectional area of the couple corresponds to the quantity of the volume

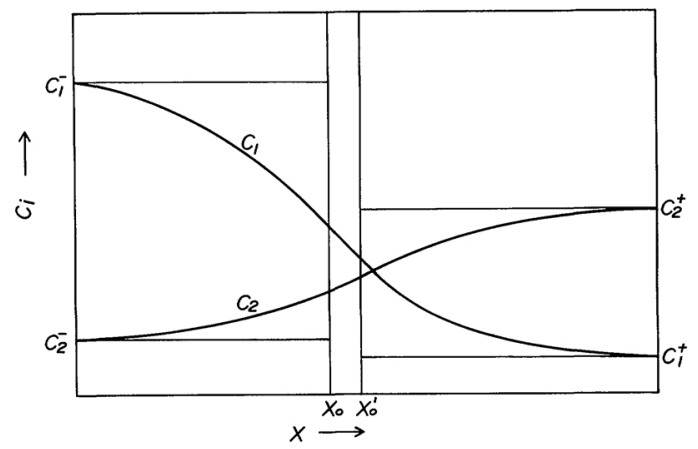

Fig. $1 C_{l}-x$ curves. The $x_{0}$ and $x_{0}^{\prime}$ satisfy eqs. (42) and (43), respectively. 
change of the couple.

For the calculation of the interdiffusion coefficients by employing Balluffi's method ${ }^{(6)}$
Son and Sano ${ }^{(11)}$ recommended to determine the positions of $x_{0}$ and $x_{0}^{\prime}$ in the couple after diffusion by use of eqs. (40) and (41)

$$
C_{2}^{+} \int_{-\infty}^{\infty}\left(x-x_{0}\right) \frac{\partial C_{1}}{\partial x} \mathrm{~d} x-C_{1}^{+} \int_{-\infty}^{\infty}\left(x-x_{0}\right) \frac{\partial C_{2}}{\partial x} \mathrm{~d} x=0
$$

and

$$
C_{2}^{-} \int_{\infty}^{-\infty}\left(x-x_{0}^{\prime}\right) \frac{\partial C_{1}}{\partial x} \mathrm{~d} x-C_{1}^{-} \int_{\infty}^{-\infty}\left(x-x_{0}^{\prime}\right) \frac{\partial C_{2}}{\partial x} \mathrm{~d} x=0
$$

When these equations are partially integrated and rearranged, the useful equations are derived as

$$
x_{0}=\frac{1}{C_{1}^{-}-C_{1}^{+} C_{2}^{-} / C_{2}^{+}} \int_{-\infty}^{\infty}\left(C_{1}-\frac{C_{1}^{+}}{C_{2}^{+}} C_{2}\right) \mathrm{d} x
$$

and

$$
x_{0}^{\prime}=\frac{1}{C_{1}^{+}-C_{1}^{-} C_{2}^{+} / C_{2}^{-}} \int_{\infty}^{-\infty}\left(C_{2}-\frac{C_{1}^{-}}{C_{2}^{-}} C_{1}\right) \mathrm{d} x .
$$

Figure 1 shows $C_{1}-x$ and $C_{2}-x$ curves, in which the latter was determined from the $C_{1}-x$ and $V-x$ curves arbitrarily drawn, the positions of the $x_{0}$ and $x_{0}^{\prime}$ being obtained by use of eqs. (42) and (43). Because ${ }_{c} J_{i}$ in eq. (14) is

$$
-\widetilde{D} \frac{\partial C_{1}}{\partial x}=\frac{1}{2 t}\left\{\left[1-C_{1}\left(\bar{V}_{1}-\bar{V}_{2}\right)\right] \int_{c_{\overline{1}}}^{c_{1}} x \mathrm{~d} C_{1}+\bar{V}_{2} C_{1} \int_{c_{\overline{1}}}^{c_{1}}\left(\frac{\bar{V}_{1}-\bar{V}_{2}}{\bar{V}_{2}}\right) x \mathrm{~d} C_{1}\right\} .
$$

Calculation of the second integrand in eq. (47) is time consuming, and especially we can not calculate the integrand in the case where multi phases diffusion occurs in a diffusion couple, because the partial molar volumes vary discontinuously at the interface between distinct phases. Consequently, eq. (46) in which the values of the partial molar volumes need not be known, is easier to use as long as $C_{i}-x$ curves are employed.

When marker movement data are available, one can use the following equation to obtain the intrinsic diffusivity relative to the volume fixed frame,

$$
D_{i}=\frac{1}{2 t} \frac{\partial x}{\partial C_{i}}\left(C_{i} X_{m}-\int_{c_{i}}^{c_{i}} x \mathrm{~d} C_{i}\right) \quad(i=1,2),
$$

$$
\text { given by }{ }^{(6)}
$$

$$
{ }_{c} J_{i}=\frac{1}{2 t} \int_{c_{\bar{i}}}^{c_{i}} x \mathrm{~d} C_{i},
$$

Fig. 1 enables us to determine ${ }_{c} J_{i}$. Combination of Table 1 and eq. (44) leads to

$$
-\tilde{D} \frac{\partial C_{1}}{\partial x}=\frac{\bar{V}_{2}}{2 t}\left(C_{2} \int_{c_{1}^{-}}^{c_{1}} x \mathrm{~d} C_{1}-C_{1} \int_{c_{2}^{-}}^{c_{2}} x \mathrm{~d} C_{2}\right)
$$

and

$$
-\frac{\tilde{D}}{V} \frac{\partial N_{1}}{\partial x}=\frac{V}{2 t}\left(C_{2} \int_{c_{\overline{1}}^{-}}^{c_{1}} x \mathrm{~d} C_{1}-C_{1} \int_{c_{\overline{2}}}^{c_{2}} x \mathrm{~d} C_{2}\right) .
$$

Starting from eq. (45), Son and Sano ${ }^{(11)}$ derived eq. (47) which corresponded to Balluffi's equation, eq. (13) in reference (6).

where $X_{m}$ is the distance from the $x_{0}$ to the Kirkendall interface. The intrinsic diffusivity for the number of mole fixed frame is given by

$$
D_{i}^{\prime}=\frac{V}{2 t} \frac{\partial x}{\partial N_{i}}\left(C_{i} X_{m}-\int_{c_{\bar{i}}}^{c_{i}} x \mathrm{~d} C_{i}\right) \quad(i=1,2)
$$

\section{Use of non-laboratory frames of reference}

If partial molar volumes depend on the composition, Fick's second law, eq. (50), does not hold.

$$
\left(\frac{\partial C_{i}}{\partial t}\right)_{x}=\left[\frac{\partial}{\partial x}\left(\tilde{D} \frac{\partial C_{i}}{\partial x}\right)_{t}\right]_{t}(i=1,2) .
$$

In order to obtain the general form of eq. (50), Cohen et al. ${ }^{(12)}$ established the conceptions of 
the new frames of reference, the so-called nonlaboratory ones, which were first outlined by Hartley and Crank $^{(13)}$. These are designated by $\xi$ and $\eta$, being defined as follows;

$$
\mathrm{d} \xi=\frac{1}{V} \mathrm{~d} x
$$

and

$$
\mathrm{d} \eta=\rho \mathrm{d} x .
$$

When the $\xi$ and $\eta$ coordinates are used instead of the actual frame $x$, the second order differential equations such as Fick's second law are given by

$$
\left(\frac{\partial N_{i}}{\partial t}\right)_{\xi}=\left[\frac{\partial}{\partial \xi}\left(\frac{\tilde{D}}{V^{2}} \frac{\partial N_{i}}{\partial \xi}\right)_{t}\right]_{t} \quad(i=1,2)
$$

and

$$
\left(\frac{\partial W_{i}}{\partial t}\right)_{\eta}=\left[\frac{\partial}{\partial \eta}\left(\rho^{2} \widetilde{D} \frac{\partial W_{i}}{\partial \eta}\right)_{t}\right]_{t} \quad(i=1,2) .
$$

Cohen et al. stated that eq. (53) was valid on the assumption of sufficiently small initial concentration difference, but the restriction has been lately eliminated ${ }^{(1)(14)}$, and eq. (53) retains in general. Arguments analogous to those in references (1) and (14) will lead to the general validity of eq. (54). With the aid of the Boltzmann Matano method ${ }^{(15)}$ eqs. (53) and
(54) can be graphically solved, when $N_{i}-\xi$ and $W_{i}-\eta$ coordinates are constructed. From eq. (53) we may determine the flux, $-(\tilde{D} / V)\left(\partial N_{i}\right)$ $\partial x$ ), which is equal to the one relative to the number of mole fixed frame of reference. The solution of eq. (54) corresponds to the mass flux, $-\rho^{2} \tilde{D}\left(\partial W_{i} / \partial \eta\right)=-\rho \tilde{D}\left(\partial W_{i} / \partial x\right)$, relative to the mass fixed frame, so that the quotient which is obtained by dividing the solution by $M_{i}$ becomes identical with the flux given by eq. (13). If the concentrations, $N_{i}$ and $W_{i}$, at the Kirkendall interface are known, we can estimate $D_{i}^{\prime}$ and $D_{i}^{\prime \prime}$ with knowledge of the distance from the Matano interface to the Kirkendall interface on the $\xi$ and $\eta$ coordinates.

The procedure to obtain the various types of the fluxes mentioned above always needs to determine the positions of the Matano interfaces on the respective frame of reference. On the contrary, the Sauer and Freise method ${ }^{(16)}$ enables us to calculate the interdiffusion coefficients without knowing the position of the Matano interface. The method is characterized by using the actual frame $x$, but it was shown by den Broeder ${ }^{(17)}$ that the method was equivalent to solving eq. (53) by the Boltzmann Matano method. The equation presented by den Broeder is

$$
-\frac{\widetilde{D}}{V} \frac{\partial N_{i}}{\partial x}=\frac{N_{i}^{-}-N_{i}^{+}}{2 t}\left[(1-Y) \int_{-\infty}^{x} \frac{Y}{V} \mathrm{~d} x+Y \int_{x}^{\infty} \frac{1-Y}{V} \mathrm{~d} x\right] \quad(i=1,2),
$$

where

$$
Y=\frac{N_{i}-N_{i}^{-}}{N_{i}^{+}-N_{i}^{-}} \quad(i=1,2) .
$$

Using $Y$, van $\operatorname{Loo}^{(18)}$ introduced the following equation to calculate the intrinsic flux,

$$
-D_{i} \frac{\partial C_{i}}{\partial x}=\frac{1}{2 t}\left(N_{i}^{-} \int_{x_{m}}^{\infty} \frac{1-Y}{V} \mathrm{~d} x-N_{i}^{+} \int_{-\infty}^{x_{m}} \frac{Y}{V} \mathrm{~d} x\right) \quad(i=1,2) .
$$

Here $x_{m}$ means the position of the Kirkendall interface on the frame $x$. From eqs. (23) and (57) one obtains

$$
-\frac{D_{i}^{\prime}}{V} \frac{\partial N_{i}}{\partial x}=\frac{1}{2 t}\left(N_{i}^{-} \int_{x_{m}}^{\infty} \frac{1-Y}{V} \mathrm{~d} x-N_{i}^{+} \int_{-\infty}^{x_{m}} \frac{Y}{V} \mathrm{~d} x\right) \quad(i=1,2) .
$$

Rearrangement of eq. (55) yields

$$
-\frac{\tilde{D}}{V} \frac{\partial N_{i}}{\partial x}=\frac{1}{2 t}\left[N_{i}^{-} \int_{x}^{\infty} \frac{1-Y}{V} \mathrm{~d} x-N_{i}^{+} \int_{-\infty}^{x} \frac{Y}{V} \mathrm{~d} x\right]+C_{i}\left[\frac{V}{2 t}\left(\int_{-\infty}^{\infty} \frac{Y}{V} \mathrm{~d} x-\int_{x}^{\infty} \frac{1}{V} \mathrm{~d} x\right)\right] \quad(i=1,2) .
$$

Using $x_{m}$ for the integration limit, $x$, in eq. (59), we find that the second bracketed term on the right hand side in eq. (59) is identical with $u^{\prime}$ in eqs. (18) and (21), because the first term on 
the right hand side in eq. (59) is equivalent to the right hand side in eq. (58).

\section{Discussion}

As being described by Cohen et al. ${ }^{(12)}$, equal increments of $\xi$ contain equal numbers of moles of solution, so that fluxes across the planes fixed with respect to the $\xi$ need to satisfy eq. (6). When we use the $N_{i}-\xi$ coordinate system, therefore, diffusion problems can be treated, as if total molar volumes of alloys do not depend on the composition. Accordingly, $D_{i}^{\prime}$ determined from eq. (58) are related to $\widetilde{D}$ by eq. (32), which has the same form as Darken ${ }^{(7)}$ has derived on the assumption of the constant total molar volume. Moreover, the moving velocity of marker relative to the $\xi$ is independent of both the volume change on mixing and difference between atomic volumes of components 1 and 2, arising from only the difference in magnitude of the fluxes of components 1 and 2 across the marker fixed plane.

Under the implicit agreement that the marker is fixed with respect to lattice plane, some authors tried to derive the relations between the self and intrinsic diffusivities; Darken ${ }^{(7)}$ and Manning ${ }^{(19)}$ introduced the relations without considering the difference in atomic volume between components 1 and 2 . Heumann ${ }^{(20)}$ treated the same problem considering the difference in atomic volume, but he did not eliminate partial molar volumes which arise from eq. (27), because he employed $D_{i}$ for the intrinsic diffusivity instead of $D_{i}^{\prime(21)}$. It is desirable to avoid using partial molar volumes, since there are some cases where inaccurate partial molar volumes are determined as previously stated by van $\mathrm{Loo}^{(18)}$. Consequently, use of eq. (55) for obtaining the interdiffusion coefficients and use of (58.) for the intrinsic ones are rational and less troublesome. Of course, eqs. (55) and (58) are simplified, if a diffusion couple consists of two kinds of pure metals or one of the couple halves is a pure metal.

The intrinsic diffusivity, $D_{i}^{\prime \prime}$, relative to the mass fixed frame of reference appears not to be so useful for diffusion in crystalline substances, but may be available for diffusion in liquid, when the diffusion fluxes relative to the mass fixed frame of reference are employed.

\section{Summary}

Considering five types of diffusion fluxes which are linearly related to one another, the authors tried to determine these fluxes. This situation allows to obtain the inter- and intrinsic diffusivities for binary diffusion, when volume changes of diffusion couples occur on mixing. The interdiffusion coefficients ordinarily defined can be correctly calculated irrespective of the type of fluxes and the concentration denotation. The magnitude of intrinsic diffusivity, however, depends on what is chosen for the concentration denotation. The useful intrinsic diffusivity is the one for the number of mole fixed frame of reference, if we discuss the relations between the self and intrinsic diffusivities in alloys.

\section{REFERENCES}

(1) M. Onishi and T. Shimozaki: to be published, Proc. of the Intern. Conf. on Diffusion in Metals and Alloys, 1982, Tihany, Hungary.

(2) J. Crank: Mathematics of Diffusion, Oxford University Press, London, (1967), p. 220.

(3) L. E. Trimble, D. Finn and A. Cosgarea, Jr.: Acta Met., 13 (1965), 501.

(4) S. Prager: J. Chem. Physics, 21 (1953), 1344.

(5) J. G. Kirkwood, R. L. Baldwin, P. J. Dunlop, L. J. Gosting and G. Kegeles: J. Chem. Physics, 33 (1960), 1505.

(6) R. W. Balluffi: Acta Met., 8 (1960), 871.

(7) L. S. Darken: Trans. AIME, 175 (1948), 184.

(8) M. Onishi and T. Shimozaki: J. Japan Inst. Metals, 46 (1982), 1 (in Japanese).

(9) J. P. Stark: Acta Met., 14 (1966), 228.

(10) C. Wagner: Acta Met., 17 (1969), 99.

(11) P. Son and T. Sano: J. Japan Inst. Metals, 41 (1977), 1113 (in Japanese).

(12) M. Cohen and C. Wagner and J. E. Reynolds: Trans. AIME, 197 (1953), 1534.

(13) G. S. Hartley and J. Crank: Trans. Faraday Soc., 45 (1949), 801.

(14) P. Son and T. Sano: Scripta Met., 8 (1974), 85.

(15) C. Matano: Japan J. Physics, 8 (1933), 109.

(16) F. Sauer and V. Freise: Z. Elektrochm., 66 (1962), 353.

(17) F. J. A. den Broeder: Scripta Met., 3 (1969), 321.

(18) F. J. J. van Loo: Acta Met., 18 (1970), 1107.

(19) J. R. Manning: Diffusion Kinetics for Atoms in Crystals, D. Van Nostrand Co., Prinston, (1968), p. 189.

(20) Th. Heumann: Z. Naturforsch., 32a (1977), 54.

(21) M. Onishi and T. Shimozaki: Trans. JIM, 24 (1983), 301. 


\section{Appendix}

The following nomenclature,

$M_{i}$ : atomic weight of $i(i=1,2)$,

$C_{i}$ : mole concentration of $i(i=1,2)$,

$\rho:$ density of alloys

$W_{i}$ : mass fraction of $i(i=1,2)$, and the relations,

$$
\rho=C_{1} M_{1}+C_{2} M_{2}
$$

and

$$
\rho W_{i}=C_{i} M_{i} \quad(i=1,2),
$$

are employed in the development.

Differentiation of eq. $(A \cdot 1)$ yields

$$
\mathrm{d} \rho=M_{1} \mathrm{~d} C_{1}+M_{2} \mathrm{~d} C_{2} .
$$

Considering the relation, $\mathrm{d} C_{2}=-\left(\bar{V}_{1} / \bar{V}_{2}\right) \mathrm{d} C_{1}$, and one obtains

$$
\mathrm{d} \rho=\frac{M_{1} \bar{V}_{2}-M_{2} \bar{V}_{1}}{\bar{V}_{2}} \mathrm{~d} C_{1} .
$$

Differentiation of eq. $(\mathrm{A} \cdot 2)$ gives

$$
\mathrm{d} W_{1}=M_{1} \mathrm{~d} C_{1}-W_{1} \mathrm{~d} \rho .
$$

Substitution of eq. (A-4) into eq. (A-5) yields

$$
\mathrm{d} W_{1}=\frac{W_{2} M_{1} \bar{V}_{2}+W_{1} M_{2} \bar{V}_{1}}{\bar{V}_{2}} \mathrm{~d} C_{1} .
$$

Eliminating $W_{i}(i=1,2)$ from eq. $(\mathrm{A} \cdot 6)$ by use of eq. $(\mathrm{A} \cdot 2)$ and employing the general relation, $C_{1} \bar{V}_{1}+C_{2} \bar{V}_{2}=1$, one obtains eq. (26). 